\title{
A Bilinear Formulation for the Motion Planning of Non-holonomic Parallel Orienting Platforms
}

\author{
Patrick Grosch and Federico Thomas
}

\begin{abstract}
This paper deals with the motion planning problem for parallel orienting platforms with one non-holonomic joint and two prismatic actuators which can maneuver to reach any three-degree-of-freedom pose of the moving platform. Since any system with two inputs and up to four generalized coordinates can always be transformed into chained form, this path planning problem can be solved using well-established procedures. Nevertheless, the use of these procedures requires a good understanding of Lie algebraic methods whose technicalities have proven a challenge to many practitioners who are not familiar with them. As an alternative, we show how by (a) properly locating the actuators, and (b) representing the platform orientation using Euler parameters, the studied path planning problem admits a closed-form solution whose derivation requires no other tools than ordinary linear algebra.
\end{abstract}

\section{INTRODUCTION}

Consider a mechanism consisting of a sphere whose center is fixed with respect to the world and whose orientation is controlled by three prismatic actuators anchored by their ends to the sphere and the world through spherical joints, as shown in Fig. 1(top). This kind of mechanism, which can be regarded as a parallel robot, have been studied by several authors due to their practical interest as a robotics wrist or, in general, as an orienting platform. The works of Innocenti and Parenti-Castelli [1], and Wohlhart [2], are usually referred as the pioneering ones on the kinematics analysis of this parallel platform.

In those applications in which it is necessary to reduce bulk, weight or cost, it is possible to substitute one of the prismatic joints by a disk that rolls without slipping with respect to the sphere as shown in Fig. 1(bottom). This idea was first introduced by Stammers in [3] and generalized by Di Gregorio [4] following the ideas presented by Grosch et al. [5].

The problem of moving the sphere between two arbitrary orientations by means of suitable movements transmitted to it by the two prismatic actuators is a challenging problem due to the non-holonomic constraint arising from the nonslip condition between the disk and the sphere. Although the experience indicates that, outside some apparent singularities, the resulting parallel robot is controllable, it is not trivial to establish such property on a mathematical basis.

This work was supported by the Spanish Ministry of Economy and Competitiveness through the Explora programme under Contract DPI201113208-E.

P. Grosch and F. Thomas are with the Institut de Robòtica i Informàtica Industrial (CSIC-UPC), Llorens Artigas 4-6, 08028 Barcelona, Spain \{pgrosch, fthomas\}eiri.upc.edu
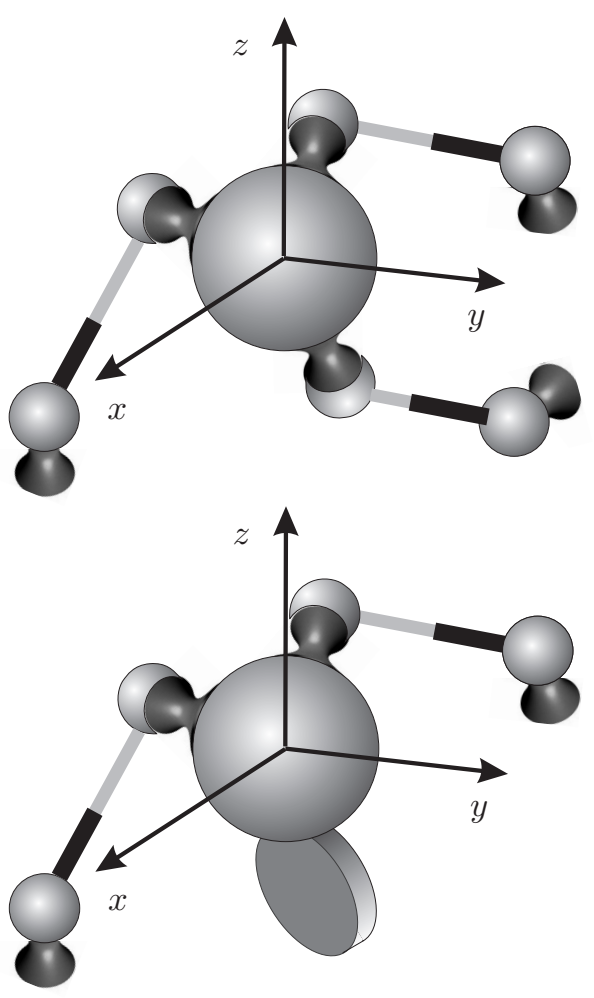

Fig. 1. Top: parallel orienting platform actuated by three prismatic joints. Bottom: non-holonomic variation in which one prismatic joint has been substituted by a disk that rolls without slipping.

If the system is analyzed at a first-order kinematic level (the dynamics of the system is not considered), it can be shown that the differential equations that describe the system can be expressed in the standard form of two-input driftless (no motion takes place for null inputs) non-holonomic system. If the dynamics of the system is introduced, the system will exhibit drift but an invertible feedback control can eliminate the dynamic parameters [6]. Therefore, the analysis of the system can be addressed as that of a twoinput driftless non-holonomic system.

An important class of non-holonomic systems for which a satisfactory understanding has been reached is the class of systems that can be put, by feedback transformation, in the so-called chained form [7]. A complete characterization of such systems (i.e., necessary and sufficient conditions for the existence of a feedback transformation to chained-form) has been provided by [8], while an algorithm for finding the necessary coordinate transform has been presented in [9]. This is important in the presented problem because it 
has been shown that a two-input driftless non-holonomic system with up to four generalized coordinates can always be transformed in chained form [10], [11], [7]. Once in chained form, different methods can be used for motion planning. Essentially two kinds of steering inputs signals have been considered: sinusoidal and piecewise constant. While the first approach was pioneered by [7], the second is attributed to [12].

It is not difficult to prove that driftless systems which, up to changes of coordinates and static or dynamic endogenous feedbacks, can be put into chained form, are necessarily flat [13]. With the flatness property, states and inputs can be parametrized by a finite set of independent variables, called the flat outputs, and their derivatives. Moreover, the number of flat outputs is equal to the number of control inputs. This nice properties are useful for motion planning because the desired trajectory can be planned in flat output space. In addition, exponential stabilizing controllers can be developed since in the flat output space, the system has the representation of a chain of integrators.

Putting a system in chained form is not an easy task and the result is not always satisfactory. The generated feedbacks introduce, in general, singularities that lead to unfeasible control inputs, i.e., infinite steering rates. Moreover, the characterization of these singularities, in the general case, is difficult due to the complexity of the generated expressions. Besides this, the standard procedures to derive chained forms assume that the number of generalized coordinates coincides with the number of degrees of freedom of the system. This leads to an important drawback when working with spatial orientations as they cannot be parameterized by only three parameters without introducing more singularities.

In this paper, it is shown how, by properly arranging the actuators and representing the platform orientation using Euler parameters, a bilinear model can be derived and this derivation requires an endogenous feedback whose singularities coincide with the mechanical singularities of the platform. Thus, no extra singularities are added. Then, it is shown how this bilinear model admits a closed-form formula for the path planning problem by relying on linear algebra arguments.

This paper is organized as follows. The kinematic model of the general parallel orienting platform with one nonholonomic joint and two prismatic actuators is derived in Section II. Next, Section III shows how this model can be expressed in bilinear form by properly arranging the actuators. This bilinear form depends on two matrices whose properties are investigated in Section IV. Then, a closed-form solution to the path planning problem is finally derived in Section V. The singularities of the system are analyzed in Section VI. A description of the experimental testbed where the derived path planner have been verified is presented in Section VII. Finally, Section VIII summarizes the main results and gives some prospects for further research.
II. KINEMATIC MODEL OF NON-HOLONOMIC PARALLEL ORIENTING PLATFORMS

\section{A. Notation}

$\mathbf{R} 3 \times 3$ rotation matrix defining the orientation of the moving platform.

$\omega$ vector of angular velocities.

$\hat{\mathbf{r}}$ unit vector of the non-holonomic constraint. Rotations about this axis are forbidden.

$\mathbf{a}_{i}$ position vector of leg attachment $i$ to the base in the base reference frame.

$\mathbf{b}_{i}^{0}$ position vector of leg attachment $i$ to the moving platform in the moving platform reference frame.

$\mathbf{b}_{i}$ position vector of leg attachment $i$ to the moving platform in the base reference frame. $\mathbf{b}_{i}=\mathbf{R} \mathbf{b}_{i}^{0}$.

$l_{i}$ length of leg $i . l_{i}=\left\|\mathbf{b}_{i}-\mathbf{a}_{i}\right\|$

$\hat{\mathbf{g}}_{i}$ unit vector in the direction of leg $i . \hat{\mathbf{g}}_{i}=\frac{\mathbf{b}_{i}-\mathbf{a}_{i}}{l_{i}}$.

\section{B. Holonomic constraints}

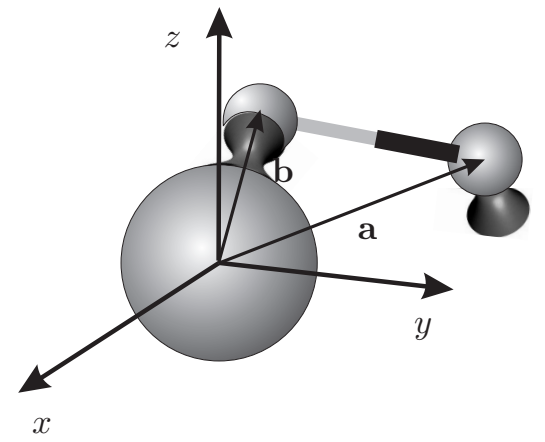

Fig. 2. A holonomic constraint is imposed on a freely rotating sphere by attaching a prismatic actuator anchored by its ends to the rotating body and the world through spherical joints.

Let us suppose a sphere that rotates $\omega_{x} \mathrm{rad} / \mathrm{s}, \omega_{y} \mathrm{rad} / \mathrm{s}$, and $\omega_{z} \mathrm{rad} / \mathrm{s}$, about the $x, y$, and $z$ axes, respectively. The linear velocity, due to these angular velocities, of a point attached to this sphere with reference position vector $\mathbf{b}$ is

$$
\begin{aligned}
v & =\left(\omega_{x}, 0,0\right)^{T} \times \mathbf{b}+\left(0, \omega_{y}, 0\right)^{T} \times \mathbf{b}+\left(0,0, \omega_{z}\right)^{T} \times \mathbf{b} \\
& =\boldsymbol{\omega} \times \mathbf{b},
\end{aligned}
$$

where $\boldsymbol{\omega}=\left(\omega_{x}, \omega_{y}, \omega_{z}\right)^{T}$. Then, the linear velocity of this point along the direction given by the unit vector $\hat{g}$ is

$$
i=\hat{\mathbf{g}} \cdot(\boldsymbol{\omega} \times \mathbf{b})=\boldsymbol{\omega} \cdot(\mathbf{b} \times \hat{\mathbf{g}}) .
$$

Now, if we introduce a prismatic actuator anchored by its ends to the rotating sphere and the world through spherical joints, as depicted in Fig. 2, one degree of freedom of the rotating sphere is constrained according to (1), where $\hat{\mathrm{g}}$ is a unit vector in the direction of the actuator and $\dot{l}$, its linear velocity. 


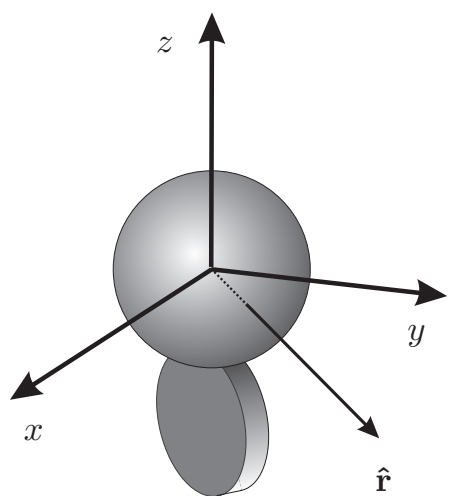

Fig. 3. A non-holonomic constraint is imposed on a freely rotating sphere by putting in contact with it a disk that freely rolls without slipping.

\section{Non-holonomic constraints}

Alternatively to the holonomic constraint introduced above, we can also constrain the motion of the freely rotating sphere by putting in contact with it a disk that rolls without slipping as shown in Fig. 3. This disk prevents the sphere to rotate about the axis oriented in the direction of the wheel. In other words,

$$
\boldsymbol{\omega} \cdot \hat{\mathbf{r}}=0
$$

\section{Constraining the motion of a sphere}

Now, let us consider the case depicted in Fig. 1(bottom) in which the rotation of the sphere is constrained by two actuated prismatic joints and a disk. In this case, the angular velocity of the sphere must satisfy the following system of equations:

$$
\left.\begin{array}{l}
l_{1}=\boldsymbol{\omega} \cdot\left(\mathbf{b}_{1} \times \hat{\mathbf{g}}_{1}\right) \\
l_{2}=\boldsymbol{\omega} \cdot\left(\mathbf{b}_{2} \times \hat{\mathbf{g}}_{2}\right) \\
0=\boldsymbol{\omega} \cdot \hat{\mathbf{r}}
\end{array}\right\}
$$

which can be expressed in matrix form as

$$
\mathbf{J} \boldsymbol{\omega}=\left(\begin{array}{c}
\dot{l}_{1} \\
\dot{l}_{2} \\
0
\end{array}\right)
$$

where

$$
\mathbf{J}=\left(\begin{array}{lll}
\mathbf{b}_{1} \times \hat{\mathbf{g}}_{1} & \mathbf{b}_{2} \times \hat{\mathbf{g}}_{2} & \hat{\mathbf{r}}
\end{array}\right)^{T} .
$$

Since $\hat{\mathbf{g}}_{i}=\left(\mathbf{b}_{i}-\mathbf{a}_{i}\right) / l_{i}$ and $\mathbf{b}_{i}=\mathbf{R} \mathbf{b}_{i}^{0}$, the above expression for $\mathbf{J}$ can be rewritten as:

$$
\mathbf{J}=\left(\begin{array}{ccc}
1 / l_{1} & 0 & 0 \\
0 & 1 / l_{2} & 0 \\
0 & 0 & 1
\end{array}\right)\left(\begin{array}{lll}
\mathbf{a}_{1} \times \mathbf{R} \mathbf{b}_{1}^{0} & \mathbf{a}_{2} \times \mathbf{R} \mathbf{b}_{2}^{0} & \hat{\mathbf{r}}
\end{array}\right)^{T}
$$

Therefore,

$$
\boldsymbol{\omega}=\mathbf{K}\left(\begin{array}{l}
i_{1} l_{1} \\
i_{2} l_{2}
\end{array}\right)
$$

where

$$
\left.\mathbf{K}=\left[\begin{array}{lll}
\mathbf{a}_{1} \times \mathbf{R} \mathbf{b}_{1}^{0} & \mathbf{a}_{2} \times \mathbf{R b}_{2}^{0} & \hat{\mathbf{r}}
\end{array}\right)^{T}\right]^{-1}\left(\begin{array}{cc}
1 & 0 \\
0 & 1 \\
0 & 0
\end{array}\right) .
$$

\section{DERIVING A BILINEAR MODEL}

Although three is the minimum number of parameters required to describe the kinematics of a rotating rigid body, every such three-dimensional parametrization of the motion is singular. This is the case of the Euler angles and the Cayley-Rodrigues parameters. Alternatively, a non-singular parameterization is possible by using four parameters. This is the case of the Euler parameters defined as

$$
\mathbf{q}=\left(\begin{array}{l}
a \\
b \\
c \\
d
\end{array}\right)=\left(\begin{array}{c}
\cos \frac{\phi}{2} \\
n_{x} \sin \frac{\phi}{2} \\
n_{y} \sin \frac{\phi}{2} \\
n_{z} \sin \frac{\phi}{2}
\end{array}\right) .
$$

where $\hat{\mathbf{n}}=\left(n_{x}, n_{y}, n_{z}\right)^{T}$ is the equivalent axis of rotation and $\phi$, the angle rotated about it. From this definition, one can easily derive the following constraint

$$
\|\mathbf{q}\|^{2}=a^{2}+b^{2}+c^{2}+d^{2}=1 .
$$

See [14] and [15] for a detailed analysis of Euler parameter and their connections with other parameterizations.

It can be shown that the rotation matrix, in terms of Euler parameters, can be expressed as

$$
\mathbf{R}=2\left(\begin{array}{ccc}
a^{2}+b^{2}-\frac{1}{2} & b c-a d & b d+a c \\
b c+a d & a^{2}+c^{2}-\frac{1}{2} & c d-a b \\
b d-a c & c d+a b & a^{2}+d^{2}-\frac{1}{2}
\end{array}\right) .
$$

If we substitute this parametrization of $\mathbf{R}$ in (8), the result is rather awkward. Nevertheless, an important simplification is attained if the anchor points of the prismatic actuators are oriented at $\pi / 2$ one from each other in their local reference frames. For example, if we set $\mathbf{a}_{1}=(1,0,0)^{T}$, $\mathbf{a}_{2}=(0,1,0)^{T}, \mathbf{b}_{1}^{0}=k \mathbf{a}_{1}$, and $\mathbf{b}_{2}^{0}=k \mathbf{a}_{2}$, the substitution of (11) in (8) yields

$$
\mathbf{K}=\frac{2 k}{\operatorname{det}(\mathbf{J})}\left(\begin{array}{c}
-r_{2}(a d+b c)+r_{3}(a c-b d) \\
r_{1}(a d+b c) \\
-r_{1}(a c-b d) \\
r_{2}(a d-b c) \\
r_{1}(-a d+b c)+r_{3}(a b+c d) \\
-r_{2}(a b+c d)
\end{array}\right),
$$

where $\mathbf{r}=\left(r_{1}, r_{2}, r_{3}\right)^{T}$. Further simplifications are still possible by properly locating the disk. For example, if we set $\mathbf{r}=\left(\frac{1}{\sqrt{2}}, \frac{1}{\sqrt{2}}, 0\right)^{T}$, then

$$
\mathbf{K}=\frac{\sqrt{2} k}{\operatorname{det}(\mathbf{J})}\left(\begin{array}{rr}
-a d-b c & a d-b c \\
a d+b c & b c-a d \\
b d-a c & -a b-c d
\end{array}\right) .
$$

Since the relationship between angular velocities and time derivatives of Euler parameters is given by

$$
\dot{\mathbf{q}}=\frac{1}{2}\left(\begin{array}{rrr}
-b & -c & -d \\
a & -d & c \\
d & a & -b \\
-c & b & a
\end{array}\right) \boldsymbol{\omega},
$$


the substitution of (7), with the expression of $\mathbf{K}$ given in (12), in (13) yields

$$
\dot{\mathbf{q}}=\left(\begin{array}{rrr}
-b & -c & -d \\
a & -d & c \\
d & a & -b \\
-c & b & a
\end{array}\right)\left(\begin{array}{rc}
-a d-b c & a d-b c \\
a d+b c & b c-a d \\
b d-a c & -a b-c d
\end{array}\right)\left(\begin{array}{l}
u_{1} \\
u_{2}
\end{array}\right)
$$

where

$$
u_{i}=\left(\frac{\sqrt{2} k l_{i}}{\operatorname{det}(\mathbf{J})}\right) i_{i}
$$

Equation (15) can be seen as a transformation in the input variables. It actually represents a local feedback transformation because both $\operatorname{det}(\mathbf{J})$ and $l_{i}$ depend on $\mathbf{q}$. Observe that this change of inputs is singular at the mechanical singularities of the platform, that is, at those configurations in which $\operatorname{det}(\mathbf{J})=0$. These singularities are studied in Section VI.

Now, let us define the transformation in the new input variables defined by

$$
\left(\begin{array}{l}
u_{1} \\
u_{2}
\end{array}\right)=\left(\begin{array}{rr}
a d+b c & b c-a d \\
b d-a c & -a b-c d
\end{array}\right)^{-1}\left(\begin{array}{rr}
-\frac{1}{\sqrt{2}} & 0 \\
0 & 1
\end{array}\right)\left(\begin{array}{l}
v_{1} \\
v_{2}
\end{array}\right)
$$

This is also a local feedback transformation because it depends on the orientation of the platform. Those configurations for which the matrix inverse in (16) is not defined are singularities introduced by this transformation. These singularities are also analyzed in Section VI where it is shown that they coincide with the mechanical singularities of the platform. With this input transformation, (14) can be rewritten as

$$
\dot{\mathbf{q}}=\left(\begin{array}{rrr}
-b & -c & -d \\
a & -d & c \\
d & a & -b \\
-c & b & a
\end{array}\right)\left(\begin{array}{rr}
-\frac{1}{\sqrt{2}} & 0 \\
\frac{1}{\sqrt{2}} & 0 \\
0 & 1
\end{array}\right)\left(\begin{array}{l}
v_{1} \\
v_{2}
\end{array}\right)
$$

or, alternatively, as

$$
\dot{\mathbf{q}}=\left(\mathbf{A} v_{1}+\mathbf{B} v_{2}\right) \mathbf{q},
$$

where

$$
\mathbf{A}=\frac{1}{\sqrt{2}}\left(\begin{array}{rrrr}
0 & 1 & -1 & 0 \\
-1 & 0 & 0 & -1 \\
1 & 0 & 0 & -1 \\
0 & 1 & 1 & 0
\end{array}\right)
$$

and

$$
\mathbf{B}=\left(\begin{array}{rrrr}
0 & 0 & 0 & -1 \\
0 & 0 & 1 & 0 \\
0 & -1 & 0 & 0 \\
1 & 0 & 0 & 0
\end{array}\right)
$$

This corresponds to the model of a driftless bilinear system with two inputs and four states, but it is not a minimal representation because the four states are not independent. They must satisfy (10). That is, $\mathbf{q} \in \mathbb{S}^{3}$ where $\mathbb{S}^{3}=\{\mathbf{x} \in$ $\left.\mathbb{R}^{3},\|\mathbf{x}\|^{2}=1\right\}$. This dependency is already implicit in (18). To make it explicit, let us derive (10) with respect to time to obtain

$$
\mathbf{q}^{T} \dot{\mathbf{q}}=0
$$

Then, by substituting (18) in (21), we have

$$
v_{1} \mathbf{q}^{T} \mathbf{A q}+v_{2} \mathbf{q}^{T} \mathbf{B} \mathbf{q}=0
$$

Since the above equation must hold for any value of $v_{1}$ and $v_{2}$, it can be concluded that $\mathbf{q}^{T} \mathbf{A q}=0$ and $\mathbf{q}^{T} \mathbf{B} \mathbf{q}=0$, but the quadratic form of a matrix is identically 0 if, and only if, the matrix is skew-symmetric, as is our case.

\section{A, B, And Rotations in $\mathbb{R}^{4}$}

Let us define

$$
\mathbf{C}=\mathbf{A B}=\frac{1}{\sqrt{2}}\left(\begin{array}{rrrr}
0 & 1 & 1 & 0 \\
-1 & 0 & 0 & 1 \\
-1 & 0 & 0 & -1 \\
0 & -1 & 1 & 0
\end{array}\right)
$$

Then, it can be checked that

$$
\mathbf{A}^{2}=\mathbf{B}^{2}=\mathbf{C}^{2}=\mathbf{A B C}=-\mathbf{I} .
$$

Hamilton called quadruples with these rules of multiplication a quaternion. Actually, (24) reproduces the celebrated formula that Hamilton carved into the stone of Brougham Bridge. Therefore, the real linear span of $\{\mathbf{I}, \mathbf{A}, \mathbf{B}, \mathbf{C}\}$ is isomorphic to the real algebra of quaternions. As with standard quaternions, (24) determines all the possible products of $\mathbf{A}, \mathbf{B}$, and $\mathbf{C}$ resulting in

$$
\begin{array}{ll}
\mathbf{A B}=\mathbf{C}, & \mathbf{B A}=-\mathbf{C}, \\
\mathbf{B C}=\mathbf{A}, & \mathbf{C B}=-\mathbf{A}, \\
\mathbf{C A}=\mathbf{B}, & \mathbf{A C}=-\mathbf{B} .
\end{array}
$$

According to (24), it can be said that $\mathbf{A}, \mathbf{B}$, and $\mathbf{C}$ behave as imaginary magnitudes. Then, it is not surprising that their matrix exponentials, defined according to the traditional power series, have simple expressions similar to Euler's formula:

$$
\begin{aligned}
& e^{\omega \mathbf{A}}=\sin (\omega) \mathbf{A}+\cos (\omega) \mathbf{I}, \\
& e^{\omega \mathbf{B}}=\sin (\omega) \mathbf{B}+\cos (\omega) \mathbf{I}, \\
& e^{\omega \mathbf{C}}=\sin (\omega) \mathbf{C}+\cos (\omega) \mathbf{I}
\end{aligned}
$$

Then, it is not either surprising to realize that $e^{\omega \mathbf{A}}, e^{\omega \mathbf{B}}$ and $e^{\omega \mathbf{C}}$ behave as rotations in four dimensions. Indeed, since the exponential of an antisymmetric matrix is an orthogonal matrix with determinant equal to +1 and unit length eigenvalues, $e^{\omega \mathbf{A}}, e^{\omega \mathbf{B}}$ and $e^{\omega \mathbf{C}}$ represent rotations.

According to Cayley's factorization, a 4D rotation matrix can always be expressed as the product of two matrices of the form

$$
\mathbf{R}^{L}\left(l_{1}, l_{2}, l_{3}, l_{4}\right)=\left(\begin{array}{rrrr}
l_{1} & -l_{2} & -l_{3} & -l_{4} \\
l_{2} & l_{1} & -l_{4} & l_{3} \\
l_{3} & l_{4} & l_{1} & -l_{2} \\
l_{4} & -l_{3} & l_{2} & l_{1}
\end{array}\right)
$$

and

$$
\mathbf{R}^{R}\left(r_{1}, r_{2}, r_{3}, r_{4}\right)=\left(\begin{array}{rrrr}
r_{1} & -r_{2} & -r_{3} & -r_{4} \\
r_{2} & r_{1} & r_{4} & -r_{3} \\
r_{3} & -r_{4} & r_{1} & r_{2} \\
r_{4} & r_{3} & -r_{2} & r_{1}
\end{array}\right),
$$


which are known as left- and right-isoclinic rotation matrices, respectively (see [16] for details on Cayley's factorization).

Now, it can observed that

$$
\mathbf{R}^{R}\left(r_{1}, r_{2}, r_{3}, r_{4}\right)=\gamma_{1} \mathbf{I}+\gamma_{2} \mathbf{A}+\gamma_{3} \mathbf{B}+\gamma_{4} \mathbf{C}
$$

where

$$
\left(\begin{array}{l}
\gamma_{1} \\
\gamma_{2} \\
\gamma_{3} \\
\gamma_{4}
\end{array}\right)=\left(\begin{array}{cccc}
1 & 0 & 0 & 0 \\
0 & -\frac{\sqrt{2}}{2} & \frac{\sqrt{2}}{2} & 0 \\
0 & 0 & 0 & 1 \\
0 & -\frac{\sqrt{2}}{2} & \frac{\sqrt{2}}{2} & 0
\end{array}\right)\left(\begin{array}{l}
r_{1} \\
r_{2} \\
r_{3} \\
r_{4}
\end{array}\right)
$$

Hence,

$$
\left(\begin{array}{l}
r_{1} \\
r_{2} \\
r_{3} \\
r_{4}
\end{array}\right)=\left(\begin{array}{cccc}
1 & 0 & 0 & 0 \\
0 & -\frac{\sqrt{2}}{2} & 0 & -\frac{\sqrt{2}}{2} \\
0 & \frac{\sqrt{2}}{2} & 0 & -\frac{\sqrt{2}}{2} \\
0 & 0 & 1 & 0
\end{array}\right)\left(\begin{array}{l}
\gamma_{1} \\
\gamma_{2} \\
\gamma_{3} \\
\gamma_{4}
\end{array}\right)
$$

Therefore, $\{\mathbf{I}, \mathbf{A}, \mathbf{B}, \mathbf{C}\}$ is a basis for right-isoclinic rotations and, as a consequence, (25)-(27) represent rightisoclinic rotations.

Now, after somewhat tedious algebraic manipulations, it can be checked that:

$$
\begin{aligned}
e^{\omega_{3} \mathbf{A}} e^{\omega_{2} \mathbf{B}} e^{\omega_{1} \mathbf{A}} & =\cos \left(\omega_{2}\right) \cos \left(\omega_{3}+\omega_{1}\right) \mathbf{I} \\
& +\cos \left(\omega_{2}\right) \sin \left(\omega_{3}+\omega_{1}\right) \mathbf{A} \\
& +\sin \left(\omega_{2}\right) \cos \left(\omega_{3}-\omega_{1}\right) \mathbf{B} \\
& +\sin \left(\omega_{2}\right) \sin \left(\omega_{3}-\omega_{1}\right) \mathbf{C} .
\end{aligned}
$$

Therefore, any arbitrary right-isoclinic rotation can be expressed as:

$$
\gamma_{1} \mathbf{I}+\gamma_{2} \mathbf{A}+\gamma_{3} \mathbf{B}+\gamma_{4} \mathbf{C}=e^{\omega_{3} \mathbf{A}} e^{\omega_{2} \mathbf{B}} e^{\omega_{1} \mathbf{A}}
$$

where

$$
\begin{aligned}
& \omega_{1}=\frac{1}{2}\left(\operatorname{atan} 2\left(\gamma_{2}, \gamma_{1}\right)-\operatorname{atan} 2\left(\gamma_{4}, \gamma_{3}\right)\right), \\
& \omega_{2}=\operatorname{atan} 2\left(\sin \left(\operatorname{atan} 2\left(\gamma_{4}, \gamma_{3}\right)\right), \cos \left(\operatorname{atan} 2\left(\gamma_{2}, \gamma_{1}\right)\right)\right), \\
& \omega_{3}=\frac{1}{2}\left(\operatorname{atan} 2\left(\gamma_{2}, \gamma_{1}\right)+\operatorname{atan} 2\left(\gamma_{4}, \gamma_{3}\right)\right) .
\end{aligned}
$$

This is the key result used in the next section to solve the path planning problem.

\section{PATH PLANNing}

Let us suppose that $v_{1}$ and $v_{2}$ are constant, then (18) becomes a linear differential equation which can be easily integrated

$$
\mathbf{q}(t)=e^{\left(v_{1} \mathbf{A}+v_{2} \mathbf{B}\right) t} \mathbf{q}_{0},
$$

where $\mathbf{q}_{0}$ represents the initial orientation of the platform.

Now, let us introduce a maneuver consisting in a sequence of actuations in which during $t_{1}$ seconds $v_{1}=k_{1}$ and $v_{2}=0$, then during $t_{2}$ seconds $v_{1}=0$ and $v_{2}=k_{2}$ and, finally, during $t_{3}$ seconds $v_{1}=k_{3}$ and $v_{2}=0$. The configuration reached, after this maneuver, by the moving platform can be expressed as

$$
\mathbf{q}_{f}=e^{\omega_{3} \mathbf{A}} e^{\omega_{2} \mathbf{B}} e^{\omega_{1} \mathbf{A}} \mathbf{q}_{0},
$$

where $\omega_{i}=k_{i} t_{i}$. Then, if we compare (39) with (33), it can be concluded that this simple maneuver permits to reach any desired configuration by finding the proper values of $\omega_{i}, i=1,2,3$. To this end, we first need to find the rightisoclinic rotation that drives the moving platform from $\mathbf{q}_{0}=$ $(a b c d)^{T}$ to $\mathbf{q}_{f}=\left(a^{\prime} b^{\prime} c^{\prime} d^{\prime}\right)^{T}$, that is, the set of parameters $r_{1}, r_{2}, r_{3}$ and $r_{4}$ that satisfies

$$
\left(\begin{array}{rrrr}
r_{1} & -r_{2} & -r_{3} & -r_{4} \\
r_{2} & r_{1} & r_{4} & -r_{3} \\
r_{3} & -r_{4} & r_{1} & r_{2} \\
r_{4} & r_{3} & -r_{2} & r_{1}
\end{array}\right)\left(\begin{array}{l}
a \\
b \\
c \\
d
\end{array}\right)=\left(\begin{array}{l}
a^{\prime} \\
b^{\prime} \\
c^{\prime} \\
d^{\prime}
\end{array}\right),
$$

which can be rewritten as

$$
\left(\begin{array}{rrrr}
a & -b & -c & -d \\
b & a & -d & c \\
c & d & a & -b \\
d & -c & b & a
\end{array}\right)\left(\begin{array}{l}
r_{1} \\
r_{2} \\
r_{3} \\
r_{4}
\end{array}\right)=\left(\begin{array}{l}
a^{\prime} \\
b^{\prime} \\
c^{\prime} \\
d^{\prime}
\end{array}\right) .
$$

Then, substituting (32) in (41), we obtain

$$
\left(\begin{array}{cccc}
a & \frac{\sqrt{2}}{2}(b-c) & -d & \frac{\sqrt{2}}{2}(b+c) \\
b & \frac{\sqrt{2}}{2}(-a-d) & c & \frac{\sqrt{2}}{2}(-a+d) \\
c & \frac{\sqrt{2}}{2}(a-d) & -b & \frac{\sqrt{2}}{2}(-a-d) \\
d & \frac{\sqrt{2}}{2}(b+c) & a & \frac{\sqrt{2}}{2}(-b+c)
\end{array}\right)\left(\begin{array}{l}
\gamma_{1} \\
\gamma_{2} \\
\gamma_{3} \\
\gamma_{4}
\end{array}\right)=\left(\begin{array}{l}
a^{\prime} \\
b^{\prime} \\
c^{\prime} \\
d^{\prime}
\end{array}\right)
$$

Solving this linear system yields

$$
\left(\begin{array}{l}
\gamma_{1} \\
\gamma_{2} \\
\gamma_{3} \\
\gamma_{4}
\end{array}\right)=\left(\begin{array}{cccc}
a & b & c & d \\
\frac{b-c}{\sqrt{2}} & -\frac{a+d}{\sqrt{2}} & \frac{a-d}{\sqrt{2}} & \frac{b+c}{\sqrt{2}} \\
-d & c & -b & a \\
\frac{b+c}{\sqrt{2}} & -\frac{a-d}{\sqrt{2}} & -\frac{a+d}{\sqrt{2}} & -\frac{b-c}{\sqrt{2}}
\end{array}\right)\left(\begin{array}{l}
a^{\prime} \\
b^{\prime} \\
c^{\prime} \\
d^{\prime}
\end{array}\right) .
$$

Finally, substituting these values of $\gamma_{1}, \ldots, \gamma_{4}$ in (35), (36), and (37), we get the values of $\omega_{1}, \omega_{2}$, and $\omega_{3}$, respectively, that define the maneuver that drives the platform from $\mathbf{q}_{0}$ to $\mathbf{q}_{f}$.

\section{Singularities}

The mechanical singularities of the studied platform are those configurations in which $\operatorname{det}(\mathbf{J})=0$. From (5), it can be concluded that they correspond to those orientations in which the vectors $\mathbf{a}_{1} \times \mathbf{b}_{1}, \mathbf{a}_{1} \times \mathbf{b}_{1}$, and $\hat{\mathbf{r}}$ lie on a plane. The expansion of (6) in terms of Euler parameters permits to formulate this geometric condition in algebraic terms as

$$
d^{2} a(b-c)+c^{2} b(a-d)-b^{2} c(a+d)-a^{2} d(b+c)=0 .
$$

The substitution of these parameters by their definition given in (9) yields

$$
(\cos \phi-1)(p \cos \phi+q \sin \phi+r)=0,
$$

where

$$
\begin{aligned}
& p=n_{x} n_{z}\left(1-n_{y}^{2}\right)+n_{y} n_{z}\left(1-n_{x}^{2}\right), \\
& q=n_{y}\left(1-n_{y}^{2}\right)-n_{x}\left(1-n_{x}^{2}\right), \\
& r=n_{x} n_{z}\left(1+n_{y}^{2}\right)+n_{y} n_{z}\left(1+n_{x}^{2}\right) .
\end{aligned}
$$


Then, the configuration is singular if, and only if, $\phi=0$, or

$$
\phi=\operatorname{atan} 2(q, p) \pm \arccos \left(\frac{-r}{\sqrt{p^{2}+q^{2}}}\right) .
$$

To derive the bilinear model presented in Section III, two input transformations are needed that might introduce extra singularities. The first input transformation (15) is only singular in a mechanical singularity, so it does not introduce any new singularity. The second input transformation (16) is apparently more complicated but the expansion of the determinant of the matrix that depends on the configuration yields

$$
-a^{2} d(b+c)-b^{2} c(a+c)+c^{2} b(d-a)+d^{2} a(c-b)=0,
$$

which is identical to (44), so it does not introduce any new singularity either.

\section{EXPERIMENTAL TESTBED}

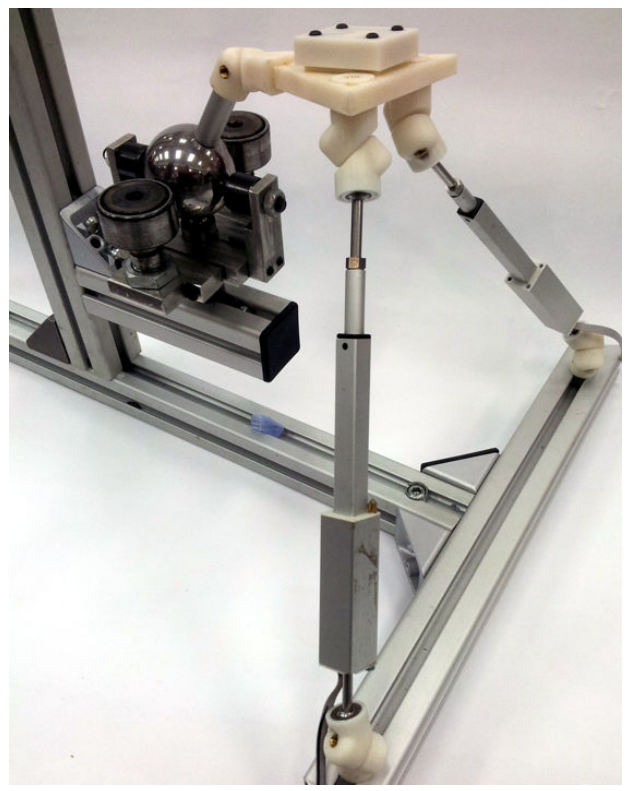

Fig. 4. Implemented experimental testbed.

The testbed shown in Fig. 4 has been implemented for validating the the presented path planner. The two prismatic actuators are miniature Firgelli linear actuators with a stroke of $100 \mathrm{~mm}$. The non-holonomic joint is based on steel ball extracted from a 3D rolling unit of a material handling system. It has been perforated with electric discharge machining. Its motion is constrained by two rollers arranged in opposing positions from the center. Three free-rolling spheres are used to keep the joint centered in the plane perpendicular to the line between the contact points of the rollers.

The two motor controllers are based on DC motor drivers (LM18200) and a PIC18f2550 with USB communication with an external PC.

The orientation of the platform is measured using a PhidgetSpatial $3 / 3 / 3$ sensor that communicates via USB with the external PC.

\section{CONCLUSION}

Designing a parallel orienting platform with only two actuators - to reduce bulk, weight or cost - becomes feasible by introducing mechanical elements that lead to nonholonomic constraints. Unfortunately, the advantages of these designs might seem dubious when facing the necessity of introducing a path planner to generate the required maneuvers to reach a target from a given initial configuration. This paper presents an alternative, based on the proper arrangement of the actuators and the parameterization of the orientation using Euler parameters, to the dominating Lie algebraic methods to design this path planner. The result is a closed-form singularity-free path planner as all introduced singularities are subsumed by the mechanical singularities of the platform. The result is a practical algorithm for planning and controlling the motions of the studied platform that can help to achieve all its potential benefits.

The presented ideas seem to be applicable to other nonholonomic mechanical systems whose orientation has to be controlled. This is a point that deserves further attention.

\section{REFERENCES}

[1] C. Innocenti and V. Parenti-Castelli, "Echelon form solution of direct kinematics for the general fully-parallel spherical wrist," Mechanism and Machine Theory, vol. 28, no. 4, pp. 553-561, 1993.

[2] K. Wohlhart, "Displacement analysis of the general spherical Stewart platform," Mechanism and Machine Theory, vol. 29, no. 4, pp. 581-589, 1994.

[3] C.W., Stammers, P.H. Prest, and C.G. Mobley, "A friction drive robot wrist: electronic and control requirements," Mechatronics, Vol. 2, No. 4, pp. 391-401, 1992.

[4] R. Di Gregorio, "Position analysis and path planning of the S-(nS)PUSPU and S-(nS)PU-2SPU underactuated wrists," ASME Journal Of Mechanisms and Robotics, Vol. 4, No. 2, 2012.

[5] P. Grosch, R. Di Gregorio, and F. Thomas, "Generation of underactuated manipulators with non-holonomic joints from ordinary manipulators," ASME Journal Mech. Robotics, Vol. 2, No. 1, 2010.

[6] A. De Luca and G. Oriolo, "Modelling and Control of Nonholonomic Mechanical Systems," in Kinematics and Dynamics of Multi-Body Systesms (J. Angeles, A. Kecskemethy Eds.), Springer Verlag, 1995.

[7] R.M. Murray and S.S. Sastry, "Nonholonomic Motion Planning: Steering Using Sinusoids," IEEE Trans. on Automatic Control, Vol. 38, pp. 700-716, 1993.

[8] R.M. Murray, "Nilpotent bases for a class of nonintegrable distributions with applications to trajectory generation for nonholonomic systems," Math. Control Signals Systems, Vol. 7, pp. 58-75, 1994.

[9] D. Tilbury, R.M. Murray, and S.S. Sastry, "Trajectory Generation for the N-Trailer Problem, Using Goursat Normal Form," IEEE Trans. on Automatic Control, Vol. 40, No. 5, pp. 802-819, 1995.

[10] H. Hermes, "Distributions and the Lie Algebras their bases can generate," Proc. American Mathematical Society, Vol. 106, No. 2, pp. 555-565, 1989.

[11] R.M. Murray, "Control of nonholonomic systems using chained forms," Fields Institute Communications, 1:219-245.

[12] G. Lafferriere, "A general strategy for computing steering controls of systems without drift," IEEE Conference on Decision and Control, Brighton, UK, pp. 1115-1120, 1991.

[13] J. Lévine, Analysis and Control of Nonlinear Systems. A Flatnessbased Approach, Springer, 2009.

[14] P.E. Nikravesh, R.A, Wehave, and O.K. Kwon, "Euler parameters in Computational Kinematics and Dynamics, Part 1," ASME J. Mech., Trans., and Auto. in Design, Vol. 107, No. 3, pp, 358-365, 1985.

[15] P.E. Nikravesh, O.K. Kwon, and R.A. Wehage, "Euler parameters in Computational Kinematics and Dynamics, Part 2," ASME J. Mech., Trans., and Auto. in Design, Vol. 107, No. 3, pp. 366-369, 1985.

[16] F. Thomas, "Approaching dual quaternions from matrix algebra," IEEE Trans. on Robotics, to appear. 